\title{
Accuracy and clinical feasibility of 3D-myocardial thickness map measured by cardiac computed tomogram
}

Oh-Seok Kwon, Jisu Lee, Sehwan Lim, Je-Wook Park, Hee-Jin Han, So-Hyun Yang, Inseok Hwang, Hee Tae Yu, Tae-Hoon Kim, Jae-Sun Uhm, Boyoung Joung, Moon-Hyoung Lee and Hui-Nam Pak ${ }^{*}$ (D)

\begin{abstract}
Background: Although myocardial thickness is an important variable for therapeutic catheter ablation of cardiac arrhythmias, quantification of wall thickness has been overlooked. We developed a software (AMBER) that measures 3D-myocardial thickness using a cardiac computed tomogram (CT) image, verified its accuracy, and tested its clinical feasibility.

Methods: We generated 3D-thickness maps by calculating wall thickness (WT) from the CT images of 120 patients' hearts and a 3D-phantom model (PhM). The initial vector field of the Laplace equation was oriented to calculate WT with the field lines derived from the 3D mesh. We demonstrate the robustness of the Laplace WT algorithm by comparing with the real thickness of 3D-PhM, echocardiographically measured left ventricular (LV) WT, and regional left atrial (LA) WT reported from previous studies. We conducted a pilot case of catheter ablation for atrial fibrillation (AF) utilizing real-time LAWT map-guided radiofrequency (RF) energy titration.
\end{abstract}

Results: AMBER 3D-WT had excellent correlations with the real thickness of the PhM $(R=0.968, p<0.001)$ and echocardiographically measured LVWT in 10 patients $(R=0.656, p=0.007)$. AMBER 3D-LAWT $(n=120)$ showed a relatively good match with 12 previously reported regional LAWT. We successfully conducted pilot AF ablation utilizing AMBER 3D-LAWT map-guided real-time RF energy titration.

Conclusion: We developed and verified an AMBER 3D-cardiac thickness map measured by cardiac CT images for LAWT and LVWT, and tested its feasibility for RF energy titration during clinical catheter ablation.

Keywords: Myocardial thickness, Computed tomogram, Software

\section{Introduction}

Heart size, bumpiness, curvature, and wall thickness (WT) are known to play important roles in the mechanisms of cardiac arrhythmias [1]. Cardiac computed tomogram (CT) imaging is an efficient, safe, and clinically validated diagnostic method for assessing the anatomic and histologic properties of the heart.

\section{*Correspondence: hnpak@yuhs.ac}

Division of Cardiology, Department of Internal Medicine, Yonsei University Health System, 50-1 Yonsei-ro, Seodaemun-gu, Seoul 03722, Republic of Korea
Nevertheless, the evaluation of WT on CT images has not been commercialized. This is mainly due to spatial resolution issues involving the segmentation of $\mathrm{CT}$ density corresponding to the myocardium and technical limitations of evaluating the thickness of complex and bumpy heart structures on a CT image acquired in the transverse section [2]. With improvements in the spatiotemporal resolution of CT images and increases in computing speed using graphic processing units, we developed a useful software to compute cardiac WT from CT images [3]. Compared to WT measured by 
echocardiogram, CT-based 3D-cardiac WT maps provide accurate anatomic localization and have no blind spots or foreshortening problems. It also provides advantages for various clinical uses, including arrhythmia interventions.

In this study, we developed software to measure cardiac WT using the Laplace equation and verified in CT images of a 3D-phantom model [4]. Both left atrial (LA) WT and left ventricular (LV) WT were measured at about 50,000 or more nodes after segmenting the cardiac wall based on Hounsfield units (HU) of cardiac $\mathrm{CT}$ images, and the entire chamber WT and regional WT were validated. Lastly, the LAWT color map was displayed on a 3D-electroanatomic map during atrial fibrillation (AF) catheter ablation, and we tested the feasibility of radiofrequency (RF) energy dosing according to local LAWT.

\section{Methods}

\section{Study design}

The study protocol adhered to the Declaration of Helsinki and was approved by the Institutional Review Board. Written informed consent was obtained from all patients (ClinicalTrials.gov; NCT 02176616).

\section{AMBER software algorithm for cardiac WT measurement}

The myocardial WT calculation consists of three steps (Fig. 1). In the first step, the threshold for each tissue automatically calculated on the histogram of the cardiac CT (64 Channel, LightSpeed Volume CT, Philips, Brilliance 63, Amsterdam, The Netherlands), and the investigator draws the guidelines for the basis of the myocardial wall extraction by semi-automatically. The second stage extracted the myocardial wall region, and the third stage solved the Laplace equation to calculate a myocardial WT.

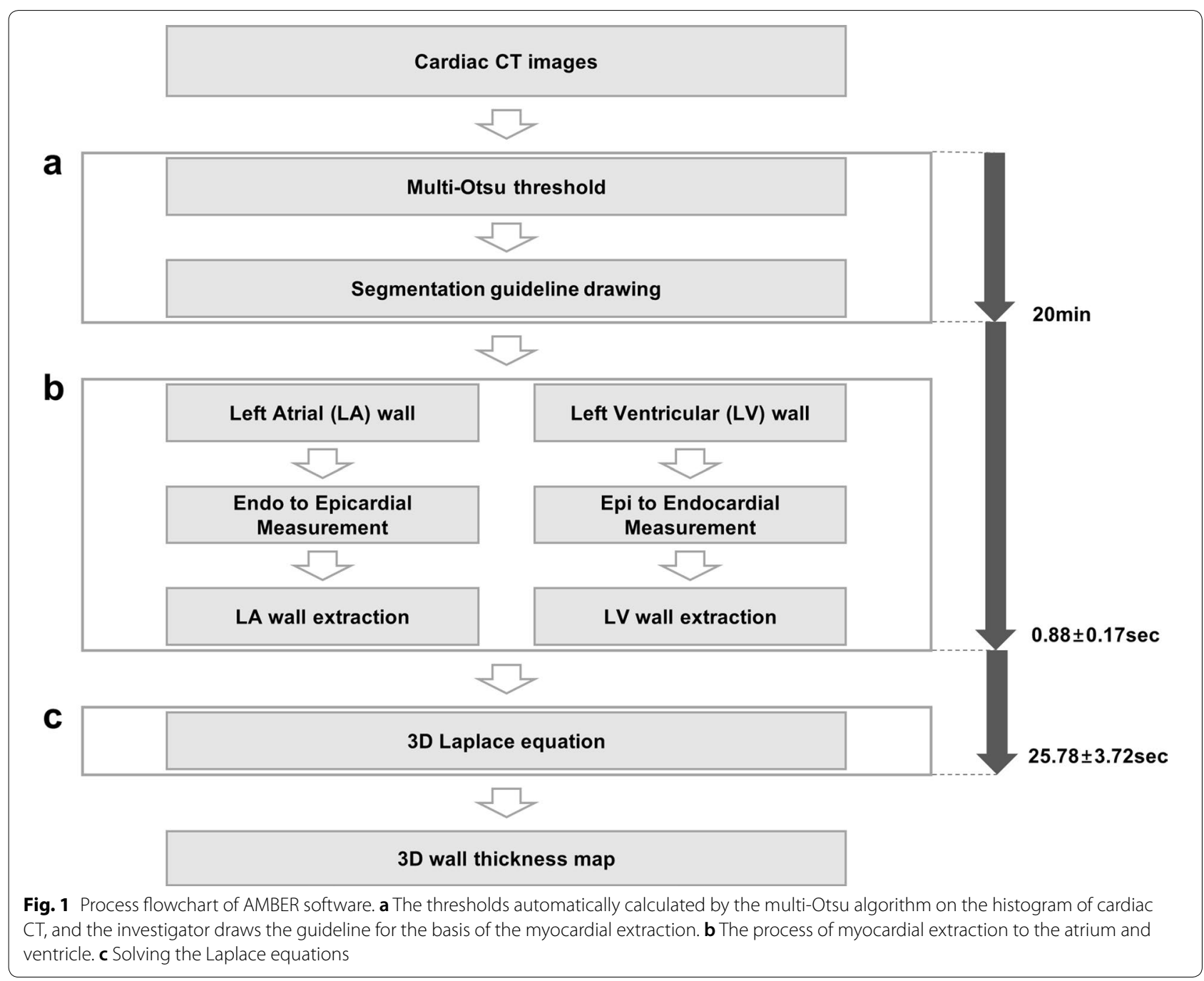




\section{Myocardium boundary segmentation in cardiac CT}

AMBER software was designed to automatically calculate the optimal endocardial boundary based on the edge protruding through the Sobel filter after removing noise with the median filter (Fig. 2a). While manually drawing the boundary line in the CT image, the multi-Otsu threshold algorithm [5] is applied in parallel to calculate the HU threshold for the density of each tissue. We assumed that cardiac CT will focus on the heart, and there will be four tissues with the highest frequency in the histogram of the HU (bone and blood pool, muscle tissue, fat, and air). Therefore, the multi-Otsu algorithm computes the histogram of the HU and the three thresholds that classify the four groups. While checking the results of the screen, the investigator selects one of the four tissue groups for detailed adjustment. For computational efficiency, all tissues of CT images were divided into 4 groups to calculate the automatically performed threshold by an independent thread running in parallel.

\section{Extraction of myocardium wall}

Extraction of cardiac wall was performed from the heart boundary line. From the boundary of endo- and epicardial surfaces obtained by the previous step, cross-overlaps with the $\mathrm{HU}$ of the myocardial tissue were extracted to the myocardial wall. In this process, two approaches were used for atrial WT and ventricular WT (Fig. 1b). For atrial WT measurement, cardiac wall was extracted by overlapping with the HU threshold of the myocardium while gradually expanding (expanding coeff $=\max (2 /$ Pixel Spacing, 8$)$ ) through morphological dilation from the endocardial boundary line (Fig. 2b). Since it progresses from the endocardium to the epicardium, the initial vector fields state for Laplace solve was determined to be 10,000 for the endocardium, 0 for the epicardium, and 5000 for the crossing area. In contrast, for ventricular WT measurement, the ventricular wall was defined from the epicardial boundary line to the endocardial surface because the ventricular endocardial surface is highly irregular due to papillary muscle and trabeculations. Since it is calculated in the opposite direction to the LA, the initial state for the Laplace solve was determined to be 10,000 at the epicardium, 0 at the endocardium, and 5000 at the intersection.

\section{Laplace equation to solve wall thickness}

The Laplace equation was applied to measure accurate cardiac WT in 3D space (Fig. 1c). The myocardial

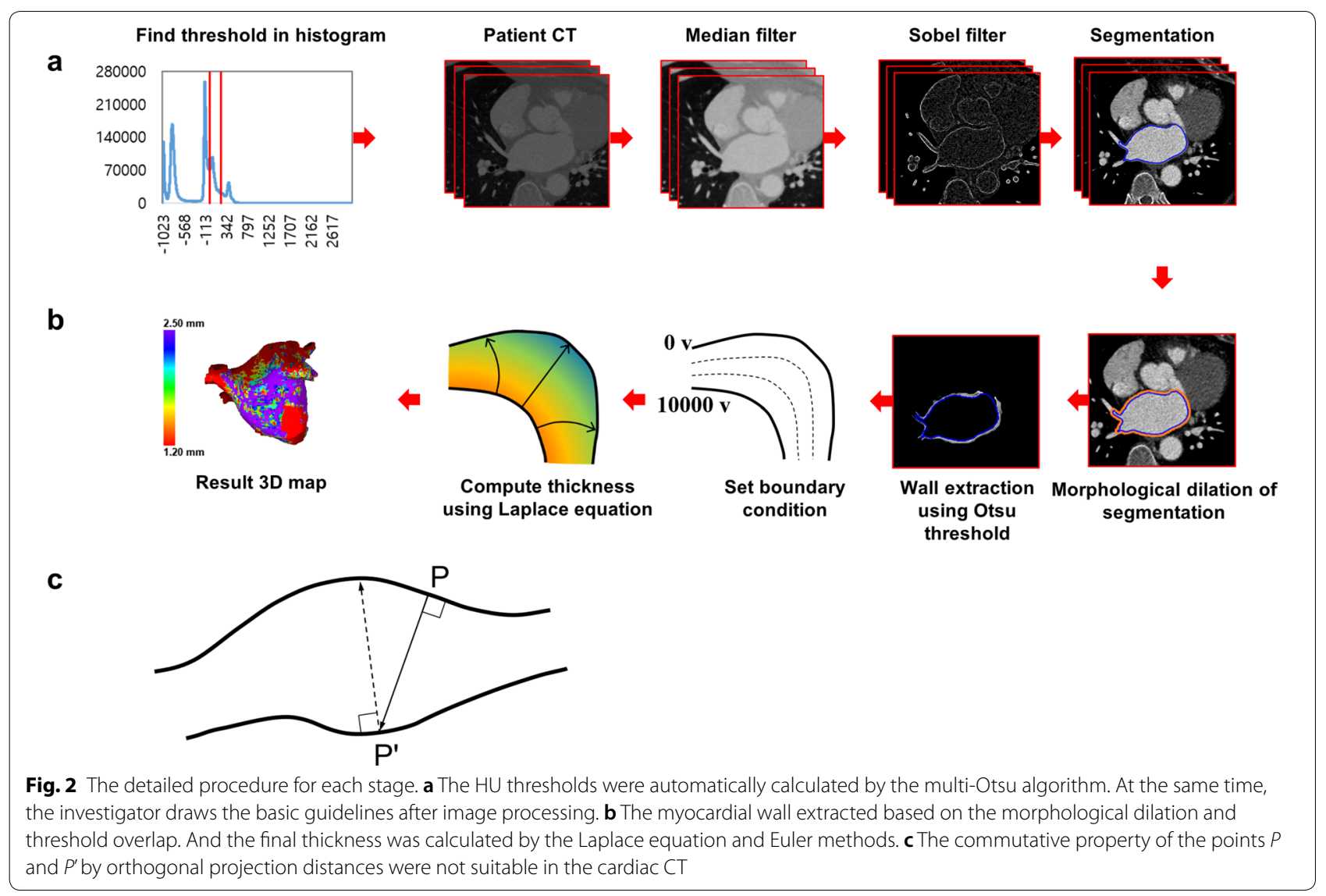


region was assumed to be topologically spherical. The orthogonal projection distance of all specific points $P \rightarrow P^{\prime}$ did not match $P^{\prime} \rightarrow P$ (Fig. 2c). Therefore, curves were connected to satisfy mutuality between the two points $P$ and $P^{\prime}$. The myocardial region was represented as the area for solving the differential equation. A vector field was formed containing a tangent vector along the field line connecting the initial vector field values set based on the boundary between the endocardium and epicardium. The Laplace equation stops when $E=10^{-5}$ or 400 iterations are met to generate an equipotential vector field. Finally, a differential equation for thickness calculation was performed by applying the Euler method of the vector field at all voxel points of the epicardium or endocardium $(d t=0.01)$.

\section{Validation of AMBER in 3D phantom model}

A 3D-phantom model was designed using FreeCAD (0.18.4) and produced with a 3D printer (output equipment: Veltz-600H, material: ABS-like, stacking thickness: $0.1 \mathrm{~mm}$ ) (Fig. 3a). The 3D-phantom model was designed to reflect the thickness of the atrium and ventricle. Two half dome regions were divided into sections with 4 different WTs each and configured with different thicknesses accounting for bidirectional measurement from inside to outside and outside to inside. Contrast enhancement is evenly distributed along the inner surface of the atrium, while ventricles have various protruding structures along the inner surface. Therefore, it was derived to measure each WT based on a uniform surface. After acquiring CT images of the 3D-phantom model (Fig. 3b), AMBER-measured 3D-WT and the computeraided design $(\mathrm{CAD})$-generated real WT were compared. a

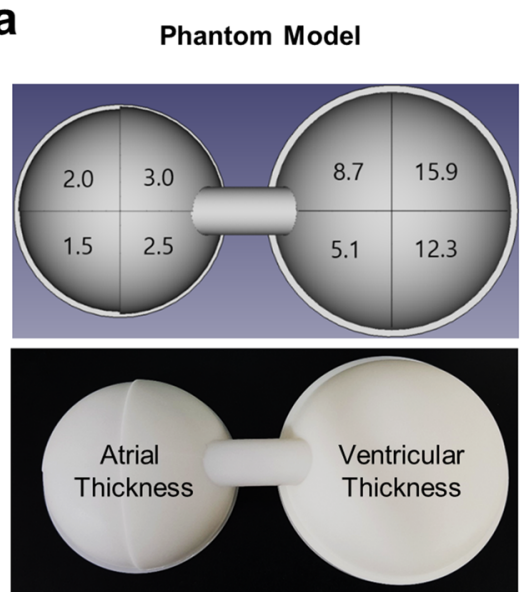

d AMBER vs Phantom model (Atrial thickness)

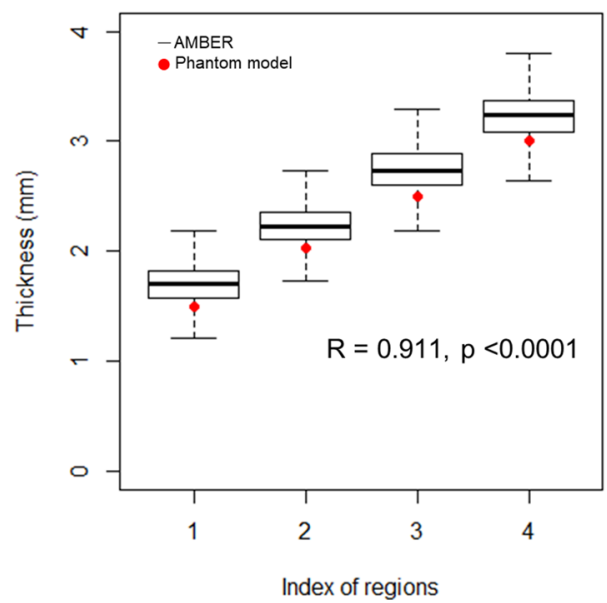

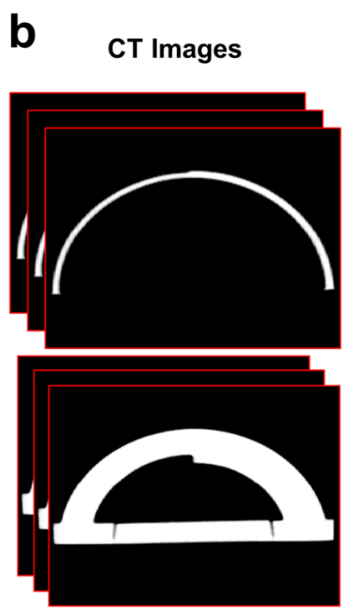

C
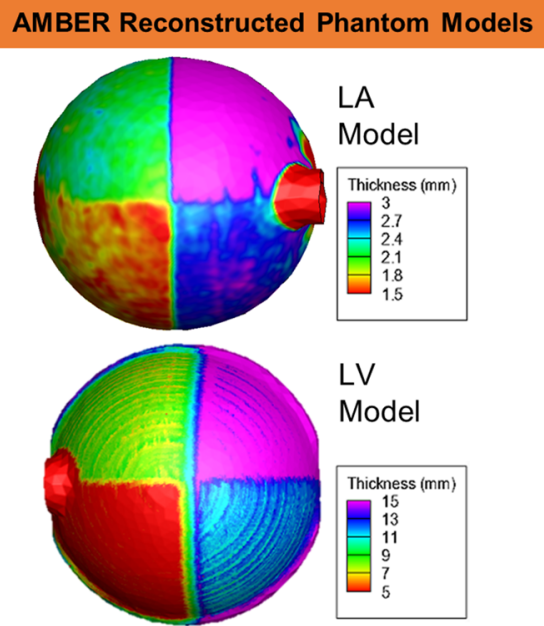

LV

Model

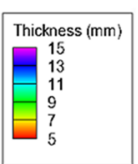

e AMBER vs Phantom model (Ventricular thickness)

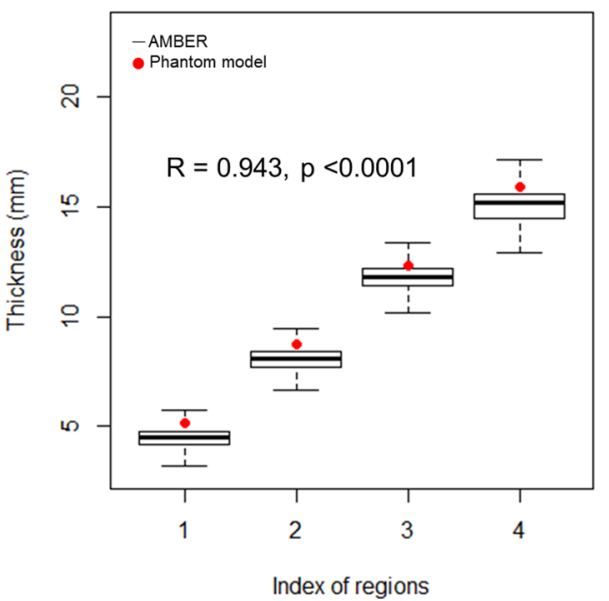

Fig. 3 Validation of Laplace wall thickness by 3D-printed phantom model. a The 3D Phantom model designed in CAD software. b The CT images of the 3D phantom model. c The reconstructed thickness of the phantom models, including atrium and ventricle. $\mathbf{d}$ Analysis of CAD thickness value and AMBER-measured atrial thickness. e Analysis of CAD thickness value and AMBER-measured ventricular thickness 
The AMBER-WT maps were analyzed except for borders where thicknesses crossed each other because these are difficult to compare between the equipotential distance of the Laplace equation and orthogonal projection distance (Fig. 3c).

\section{Validation of AMBER-measured atrial WT}

Since it is impossible to measure real atrial WT in a living human atrium, LAWT measured by AMBER in 120 patients with AF was compared with regional LAWT in 12 previously published studies (Fig. 4) [6-17]. The LAWT was measured using the Laplace equation and Euler method described above using a 3D-AMBER map composed of about 90,000 nodes. To measure by region, the LA mesh was created using the marching cube algorithm for the AMBER segmentation information. LA was divided into 8 regions based on criteria that were previously used in CT imaging [18]. Using custom made software (CUVIA, Model: SH01, ver. 2.0; Laonmed Inc., Seoul, Korea), the dividing lines of the regions were displayed on the surface of the LA mesh $[18,19]$. Based on these lines, a search algorithm was used to assign the number of each LA section to each node, and a quantitative thickness result corresponding to each region was exported (Fig. $4 \mathrm{a}-\mathrm{c}$ ). In the previous research on human LAWT, 516 studies were found based on the search keywords "human, atrium, and wall thickness," and the data were collected by selecting 12 papers that presented human LAWTs by region. If the category by region of LA was different from this study, the location of the region presented in the paper was evaluated and the LAWT value of the region most similar was selected. The overall distribution of regional LAWT presented in a total of 12 studies and 120 AF patients in this study was compared (Fig. 4d).

\section{Validation of AMBER-measured ventricular WT}

The robustness of the new software was demonstrated by comparing AMBER-measured LVWT with WT measured by echocardiogram. The LVWTs in 16 standard segments [20] were evaluated and compared in 10 patients with both cardiac CT images and standard echocardiographic images (Fig. 5a). Echocardiographic parameters were obtained according to the American Society of Echocardiography guidelines [21]. For AMBER-measured LVWT analysis, a regional LV mesh model was generated by fitting CT images of each patient to 16 standard segments of echocardiogram at the QRS gating state. The output of AMBER-measured LVWT for 16 segments was compared with echocardiographically measured LVWTs of each segment.

\section{Feasibility test for AMBER-WT map in clinical AF catheter ablation}

We tried to verify clinical feasibility of AMBER-measured WT map during AF catheter ablation procedure for a patient. Electrophysiological mapping and radiofrequency catheter ablation have been described previously. Briefly, an open irrigated-tip catheter (Smart-touch [Johnson \& Johnson Inc., Diamond Bar, CA, USA]; $30-35 \mathrm{~W}, 47^{\circ} \mathrm{C}$, contact force $>10-20 \mathrm{~g}$ ) was used to deliver radiofrequency energy for the ablation under $3 \mathrm{D}$
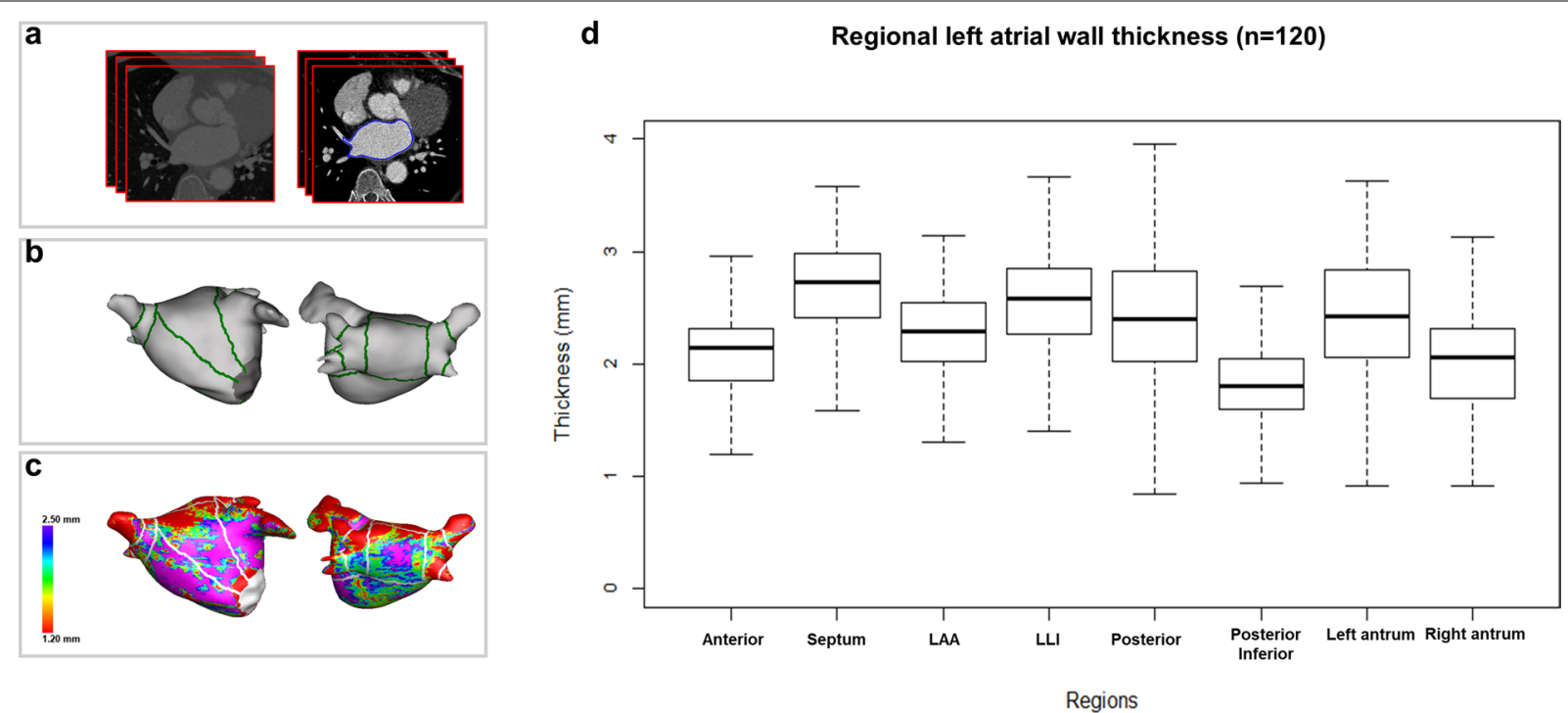

Fig. 4 Comparison analysis with 12 previously published studies. a The cardiac CT of 120 patients. b The mesh of LA segmented into 8 regions. $\mathbf{c}$ The LAWT map divided regionally. $\mathbf{d}$ The box plot of regional left atrial wall thickness of 120 patients 


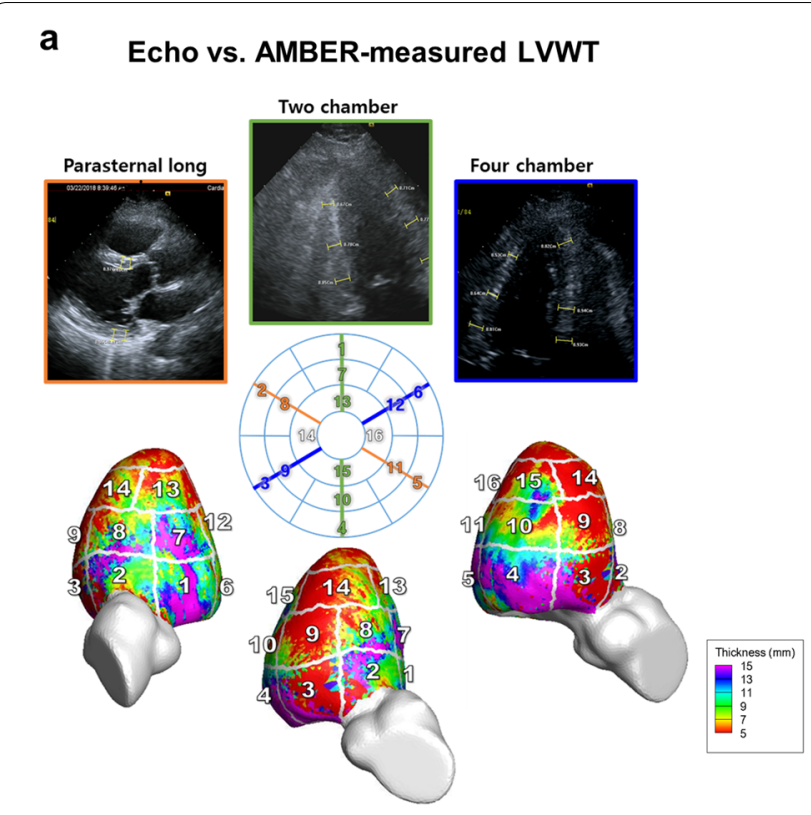

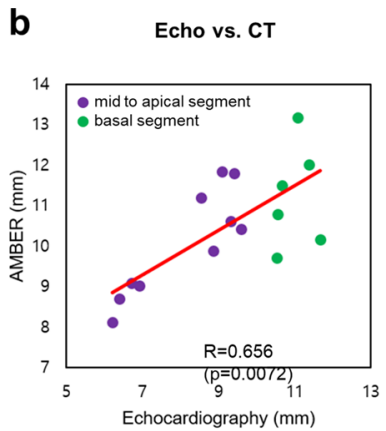

d LVWT at Mid-ventricular Segments

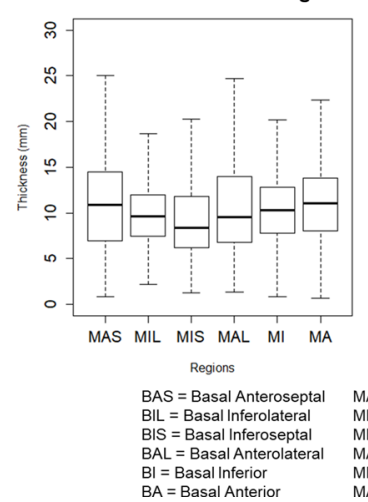

C

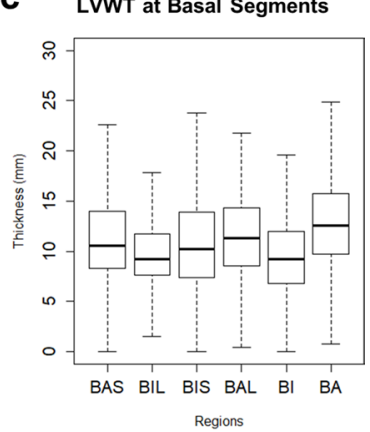

e
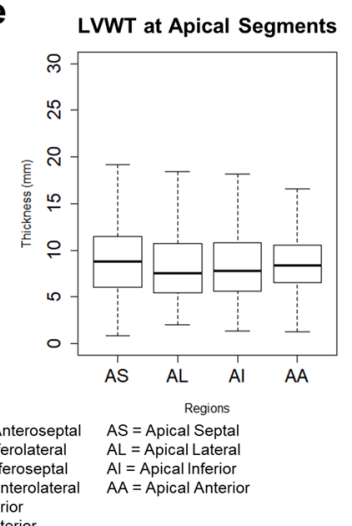

Fig. 5 The comparison results of LVWT measured by echocardiography and AMBER in 10 patients. a The echocardiography and LVWT were evaluated by splitting into a 16-segment model of the left ventricle (without apical cap). b LVWT positively correlated with echocardiography, and the lines represent the linear regression analysis. c-e Box plot evaluated divided into Basal, Mid-ventricular, and Apical segments

electroanatomic mapping (CARTO3, Johnson \& Johnson Inc. USA.) merged with 3D spiral CT. During circumferential pulmonary vein isolation (CPVI), the LAWT color map displayed in real-time on the 3D-mapping screen to confirm whether radiofrequency energy titration was possible by titrating the ablation index [22] depending on the local LAWT of the corresponding ablation site. After CPVI, bidirectional block was confirmed. The procedure was completed when there was no immediate recurrence of AF after cardioversion with an isoproterenol infusion (5-10 $\mu \mathrm{g} / \mathrm{min}$ depending on $ß$-blocker use, target sinus heart rate $120 \mathrm{bpm})$.

\section{Statistical analysis}

Because the trial was conducted as a pilot study, the sample size was driven by the computation time and feasibility of recruiting patients. Patient enrollment was open for 6 months for other clinical trials of computational modeling (CUVIA-AF2, clinical trial.gov. NCT 02558699). Results are expressed as mean values \pm standard deviation for continuous variables, and absolute number and percentages for categorical variables. Continuous variables were compared using Student's $t$ test, and categorical variables were compared using either Chi-square test or Fisher's exact test as appropriate. Comparisons of AMBER-measured WT and real WT or echocardiographically measured WT were evaluated by linear correlation methods. A $p$ value of $<0.05$ was considered statistically significant. All statistical analyses were performed using SPSS version 18.0 software (SPSS Inc., Chicago, IL, USA) and the R package (3.1.0, R Foundation for Statistical Computing, Boston, Massachusetts, United States).

\section{Results}

\section{Validation of WT map in a 3D-phantom model}

The 3D-phantom model produced by 3D-printing after computer-aided design (CAD) was used as the first verification step of the AMBER-WT software. The real thickness of the $3 \mathrm{D}$-phantom model and the virtual thickness obtained with the AMBER-WT map from acquired CT images were compared at 53,715 points. The CAD-based 3D phantom model was made into a hemispherical shape of atrial WT area $(1.5-3.0 \mathrm{~mm})$ and ventricular WT area (5.1-15.9 mm), and each hemisphere was divided into four sections of different thickness (Fig. 3a). After acquiring the 3D mesh of the phantom model, it segmented into 8 regions (Fig. 3b), and then a regionally divided WT 
map reconstructed as shown in Fig. 3c. AMBER quantified virtual WT at the atrial area of the phantom model (23,178 points) showed a good correlation with the real thickness of the phantom model $(R=0.911, p<0.0001$, Fig. 3d). The AMBER calculated WT of the ventricular area phantom model also showed a relatively good correlation with real WT $(30,537$ points, WT $R=0.943$, $p<0.0001$, Fig. 3e).

\section{Validation of AMBER-measured regional LAWT compared with previous reports}

LAWT measured by AMBER in 120 patients with AF (male 68.3\%, 60.0 \pm 10.9 years old, Table 1) was compared with LA regional WT in 12 previously published studies. The LAWT measured in cardiac CT images of 120 patients was $2.10 \pm 0.36 \mathrm{~mm}$, and the distribution at 8 different regions of LA was between 1.80 and $2.67 \mathrm{~mm}$ $(35,559 \pm 5646$ points in each patient, Fig. 4$)$. The overall LAWT distribution tended to be very similar to those of the previous 12 studies (Table 2). LAWT around the pulmonary vein area was thicker than that reported in a previously published study, but this is presumed to be due to differences in measuring sites depending on the distance from the pulmonary vein ostial level.

\section{Table 1 Baseline clinical characteristics}

\begin{tabular}{ll}
\hline & $\begin{array}{l}\text { Overall } \\
(\boldsymbol{N}=\mathbf{1 2 0})\end{array}$ \\
\hline Age, years & $60.0 \pm 10.9$ \\
Male, $n$ (\%) & $82(68.3)$ \\
AF duration, median (IQR) & $24(12-48.5)$ \\
Comorbidities, $n$ (\%) & \\
Heart failure & $26(21.7)$ \\
Hypertension & $62(51.7)$ \\
Diabetes mellitus & $25(20.8)$ \\
Stroke & $15(12.5)$ \\
Vascular disease & $7(5.8)$ \\
CHA ${ }_{2}$ DS ${ }_{2}$-VASc score & $1.97 \pm 1.6$ \\
AAD use prior to the ablation, $n(\%)$ & \\
Class IC & $54(45.0)$ \\
Class III & $64(53.3)$ \\
Echocardiographic parameters & \\
LA dimension, mm & \\
LA volume index, ml/m ${ }^{2}$ & $44.09 \pm 5.6$ \\
LV ejection fraction, \% & $42.00 \pm 11.9$ \\
E/Em & $60.52 \pm 7.3$ \\
\hline AAD, antar & $10.06 \pm 4.7$ \\
\hline
\end{tabular}

$A A D$, antiarrhythmic drug; $L A$, left atrium; $L V$, left ventricle; $E / E m$, mitral inflow velocity/mitral annulus tissue velocity

\section{Validation of AMBER-measured regional LVWT compared} with echocardiogram

Echocardiographically measured LVWT and AMBER quantified LVWT were compared in 16 segments of each LV in 10 patients (all AF patients, $70 \%$ male, $59.82 \pm 8.26$ years old) (Fig. $5 \mathrm{a}$ ). Because the endocardial side of the LV includes rugged structures such as papillary muscles and trabeculations, we measured LVWT based on the epicardial surface of the CT image as a reference. The LVWT of 16 segments measured at QRS gated timing showed a significant correlation between AMBERCT and echocardiogram overall $(R=0.656, p=0.007$, Fig. 5b). In general, AMBER-measured regional LVWT tended to be thicker than echocardiographically measured LVWT. This difference was more pronounced in the thicker segments of the LV those included papillary muscle (Fig. 5b). As shown in Fig. 5c-e, AMBER-measured LVWT was relatively thinner in the basal to mid inferior wall segments that did not include papillary muscle than in another areas.

\section{LAWT map-guided AF catheter ablation}

We used AMBER-measured 3D LAWT color map for clinical AF catheter ablation in a patient. The preobtained cardiac CT scan was used to analyze the AMBER 3D LAWT map in advance the day before the procedure (processing time 20-30 min). During the procedure, a 3D electroanatomic map was generated by endocardial bipolar electrograms ( $>500$ points) acquired with a multi-electrode catheter, and the spatiotemporal information of each electrogram was merged with a previously analyzed LAWT map (merging time $15 \mathrm{~min}$ ). The operator performed circumferential pulmonary vein isolation on the AMBER-LAWT-merged electroanatomic map and titrated the radiofrequency power or energy delivery duration on each ablation point depending on regional LAWT (Fig. 6). Utilization of the AMBER-measured LAWT map during ablation is expected to help with effective transmural radiofrequency lesion formation while avoiding unnecessary ablation and reducing the risk of complications.

\section{Discussion}

\section{Main findings}

In this study, we developed an algorithm to measure cardiac WT from CT images using Laplace equations and image processing techniques. The accuracy of this algorithm for WT measurement was verified by comparing with the $3 \mathrm{D}$-phantom model. In addition, regional LAWTs acquired from 120 patients with AF were compared with the results of previous studies. The regional LVWTs of 10 patients were compared with the WT 
Table 2 Comparison of regional left atrial wall thickness and 12 previously reported studies

\begin{tabular}{|c|c|c|c|c|c|c|c|c|c|}
\hline & LA overall & Anterior wall & Septum & $\begin{array}{l}\text { LA } \\
\text { Appendage }\end{array}$ & $\begin{array}{l}\text { Left lateral } \\
\text { isthmus area }\end{array}$ & Posterior wall & $\begin{array}{l}\text { Posterior } \\
\text { inferior wall }\end{array}$ & $\begin{array}{l}\text { Right antral } \\
\text { area }\end{array}$ & $\begin{array}{l}\text { Left antral } \\
\text { area }\end{array}$ \\
\hline \multicolumn{10}{|l|}{$\begin{array}{l}\text { Histology- } \\
\text { based LAWT }\end{array}$} \\
\hline $\begin{array}{l}\text { Hall et al. [8] } \\
\quad(n=34)\end{array}$ & - & $1.86 \pm 0.59$ & $2.20 \pm 0.82$ & - & $1.60 \pm 0.48$ & $1.06 \pm 0.49$ & $1.40 \pm 0.46$ & - & - \\
\hline $\begin{array}{l}\text { Platonov } \\
\text { et al. [15] } \\
(n=59)\end{array}$ & - & - & - & - & - & $2.60 \pm 1.00$ & - & - & - \\
\hline $\begin{array}{l}\text { Yen Ho } \\
\quad \text { et al. [17] } \\
\quad(n=26)\end{array}$ & - & $3.30 \pm 1.20$ & $4.50 \pm 0.60$ & - & $3.90 \pm 0.70$ & - & $4.10 \pm 0.70$ & - & - \\
\hline \multicolumn{10}{|l|}{$\begin{array}{l}\text { CT image- } \\
\text { based LAWT }\end{array}$} \\
\hline $\begin{array}{l}\text { Imada } \\
\quad \text { et al. [11] } \\
\quad(n=17)\end{array}$ & - & 2.60 & - & - & - & - & - & - & - \\
\hline $\begin{array}{l}\text { Hoffmeister } \\
\text { et al. [10] } \\
(n=42)\end{array}$ & $2.40 \pm 0.50$ & - & - & - & - & - & - & - & - \\
\hline $\begin{array}{l}\text { Pan et al. [14] } \\
\quad(n=57)\end{array}$ & - & $2.50 \pm 0.70$ & - & - & - & $1.50 \pm 0.30$ & - & - & - \\
\hline $\begin{array}{l}\text { Nakamura } \\
\text { et al. [13] } \\
(n=62)\end{array}$ & $2.40 \pm 0.20$ & - & - & - & - & - & - & - & - \\
\hline $\begin{array}{l}\text { Dewland } \\
\text { et al. [7] } \\
(n=98)\end{array}$ & 0.70 & - & - & - & - & - & - & - & - \\
\hline $\begin{array}{l}\text { Beinart et al. } \\
\quad[6](n=64)\end{array}$ & - & - & - & - & $2.10 \pm 0.63^{i}$ & $1.43 \pm 0.44^{\mathrm{ii}}$ & $1.81 \pm 0.44^{\mathrm{iii}}$ & - & - \\
\hline $\begin{array}{r}\text { Hayashi et al. } \\
{[9](n=17)}\end{array}$ & - & - & - & - & $2.38 \pm 0.36^{i v}$ & $1.44 \pm 0.17^{\vee}$ & $1.64 \pm 0.25^{\mathrm{vi}}$ & - & - \\
\hline $\begin{array}{l}\text { Takahashi } \\
\text { et al. [16] } \\
(n=50)\end{array}$ & - & $1.65 \pm 0.44$ & $1.17 \pm 0.29$ & $1.14 \pm 0.40$ & - & $1.61 \pm 0.31$ & - & $0.66 \pm 0.12^{\mathrm{vii}}$ & $0.65 \pm 0.16^{\text {viii }}$ \\
\hline $\begin{array}{l}\text { Inoue et al. } \\
{[12]} \\
(n=86)\end{array}$ & $1.50 \pm 0.50$ & - & - & - & - & - & $1.60 \pm 0.50^{\mathrm{ix}}$ & $1.60 \pm 0.50^{x}$ & $1.40 \pm 0.50^{\mathrm{xi}}$ \\
\hline \multicolumn{10}{|l|}{$\begin{array}{l}\text { AMBER-based } \\
\text { LAWT }\end{array}$} \\
\hline \multicolumn{10}{|l|}{$\begin{array}{c}\text { Overall } 120 \\
\text { patients }\end{array}$} \\
\hline $\begin{array}{c}\text { Bipolar Volt- } \\
\text { age, mV }\end{array}$ & $1.96 \pm 0.70$ & $1.73 \pm 0.81$ & $1.65 \pm 0.67$ & $2.51 \pm 1.10$ & $1.49 \pm 0.86$ & $2.22 \pm 1.13$ & $1.84 \pm 0.81$ & - & - \\
\hline LAWT, mm & $2.10 \pm 0.36$ & $2.08 \pm 0.36$ & $2.67 \pm 0.50$ & $2.30 \pm 0.39$ & $2.57 \pm 0.58$ & $2.40 \pm 0.67$ & $1.80 \pm 0.42$ & $2.01 \pm 0.47$ & $2.40 \pm 0.59$ \\
\hline \multicolumn{10}{|l|}{$\operatorname{PeAF}(n=69)$} \\
\hline $\begin{array}{l}\text { Bipolar Volt- } \\
\text { age, mV }\end{array}$ & $1.93 \pm 0.72$ & $1.76 \pm 0.86$ & $1.61 \pm 0.68$ & $2.49 \pm 1.13$ & $1.49 \pm 0.88$ & $2.15 \pm 1.06$ & $1.70 \pm 0.83$ & - & - \\
\hline LAWT, mm & $2.12 \pm 0.37$ & $2.10 \pm 0.38$ & $2.67 \pm 0.50$ & $2.33 \pm 0.42$ & $2.59 \pm 0.61$ & $2.42 \pm 0.69$ & $1.78 \pm 0.38$ & $1.98 \pm 0.48$ & $2.39 \pm 0.57$ \\
\hline \multicolumn{10}{|l|}{$\begin{array}{c}\text { PeAF \& PAF } \\
(n=38)\end{array}$} \\
\hline $\begin{array}{l}\text { Bipolar Volt- } \\
\text { age, mV }\end{array}$ & $2.01 \pm 0.65$ & $1.69 \pm 0.75$ & $1.72 \pm 0.64$ & $2.53 \pm 0.99$ & $1.46 \pm 0.83$ & $2.38 \pm 1.18$ & $2.05 \pm 0.75$ & - & - \\
\hline LAWT, mm & $2.06 \pm 0.36$ & $2.04 \pm 0.36$ & $2.65 \pm 0.47$ & $2.23 \pm 0.35$ & $2.51 \pm 0.57$ & $2.32 \pm 0.67$ & $1.81 \pm 0.47$ & $2.05 \pm 0.48$ & $2.40 \pm 0.64$ \\
\hline \multicolumn{10}{|l|}{$\operatorname{PAF}(n=9)$} \\
\hline $\begin{array}{l}\text { Bipolar Volt- } \\
\text { age, mV }\end{array}$ & $2.08 \pm 0.69$ & $1.79 \pm 0.58$ & $1.84 \pm 0.69$ & $2.73 \pm 1.23$ & $1.41 \pm 0.72$ & $2.46 \pm 1.37$ & $1.99 \pm 0.70$ & - & - \\
\hline LAWT, mm & $2.21 \pm 0.21$ & $2.24 \pm 0.18$ & $2.88 \pm 0.46$ & $2.41 \pm 0.21$ & $2.74 \pm 0.35$ & $2.58 \pm 0.53$ & $2.09 \pm 0.33$ & $2.03 \pm 0.31$ & $2.62 \pm 0.44$ \\
\hline
\end{tabular}




\section{Table 2 (continued)}

$\mathrm{LA}$, left atrium; CT, computed tomography; LAWT, left atrial wall thickness; AF, atrial fibrillation; PeAF, persistent atrial fibrillation; PAF, paroxysmal atrial fibrillation In the previous study, if the region was divided into sub-regions, a similar region was selected. The selected regions are as follows: (i) Left lateral ridge; (ii) Middlesuperior posterior; (iii) Middle floor; (iv) Mitral Isthmus; (v) Middle Posterior; (vi) Middle floor; (vii) Posterior right superior PV-LA junction; (viii) Anterior left superior; PV-LA junction; (ix) Right floor; (x) right low anterior PV antrum; (xi) Left low anterior PV antrum
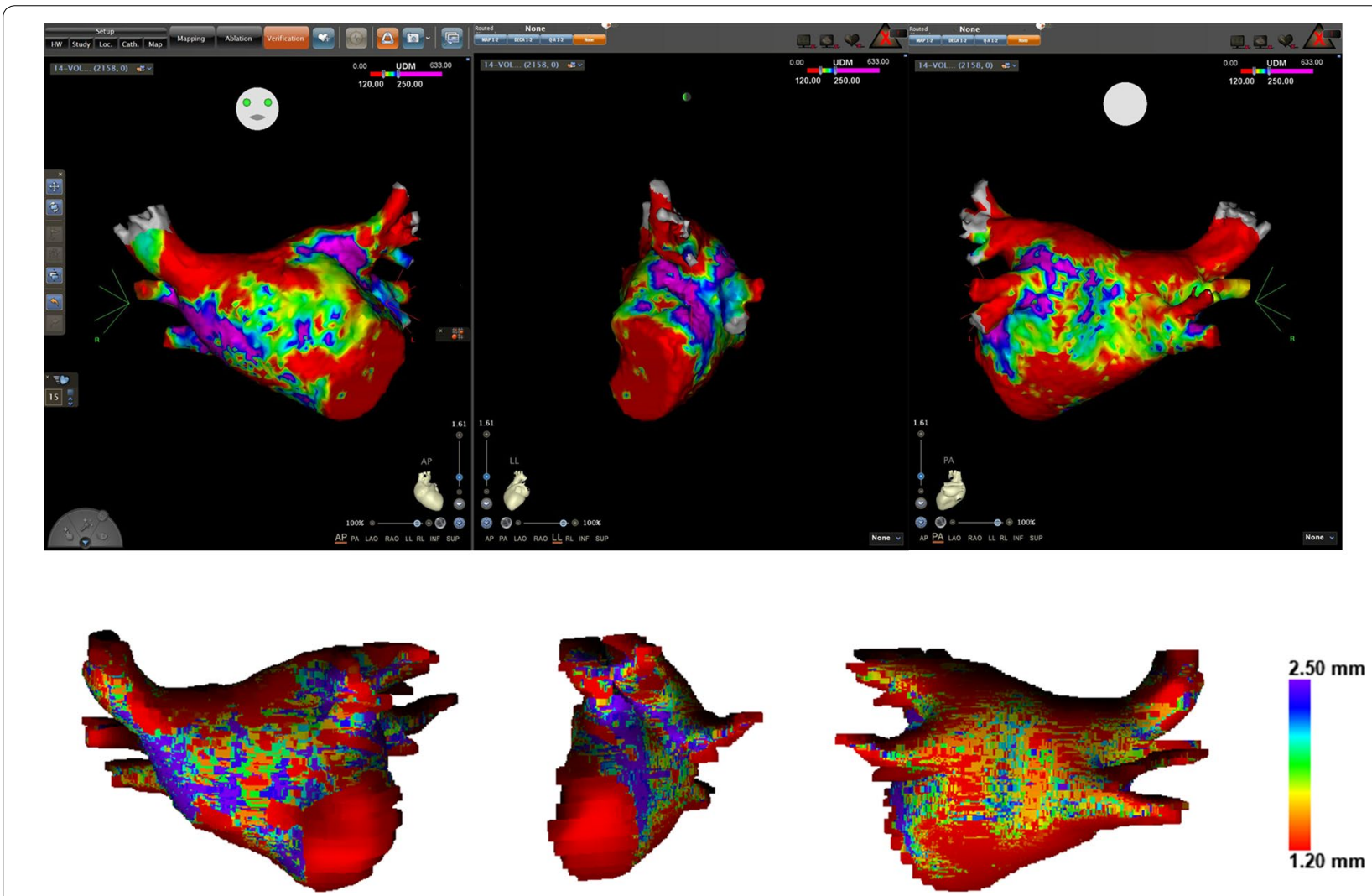

Fig. 6 During the ablation procedure, LAWT was displayed on the CARTO system. The 3D LAWT map calculated with the AMBER software (bottom) was displayed on the CARTO system (top)

measured by echocardiography to evaluate the potential for clinical applications. Finally, in a case of AF catheter ablation, the AMBER-LAWT map was displayed in realtime during CPVI to test its applicability in radiofrequency energy titration.

\section{Role of myocardial wall thickness in arrhythmogenesis}

Various animal experiments have proven that myocardium thickness affects electrical conduction and arrhythmia generation [23, 24]. The association between risk of ventricular hypertrophy and sudden death has also been demonstrated in human hearts [25]. In particular, the mid-myocardial layer of the ventricle has a long refractory period, which causes dispersion of repolarization [26]. In disease conditions, endo-epicardial dissociation and transmural reentry play an important role in the initiation and maintenance of ventricular arrhythmias, such as phase 2 reentry of Brugada syndrome, arrhythmogenic right ventricular cardiomyopathy, or non-ischemic VT.

The atrium has relatively thin myocardial WT compared to the ventricle, so the role of atrial WT on the mechanism of arrhythmia was considered negligible. However, epicardial structures such as the ligament of Marshall were shown to play a significant role in AF generation with electrical dissociation to the endocardium [27]. Recently, activation differences between epicardial breakthrough and endocardial activation were reported in surgical mapping of AF patients with structural heart diseases [28]. Anatomic and functional transmural dissociations of electrical activation and intramural reentry were demonstrated as a mechanism of AF by explant 
heart optical mapping [29] and simultaneous endo- and epicardial high density mapping in the area of thick atrial WT [30]. However, since most atrial transmural mapping studies have been conducted in the appendage area, which has many trabeculations, it is unclear how much atrial WT affects the mechanism of AF. In this study, we developed and validated a software algorithm that reconstructs atrial and ventricular WTs in three dimensions by reconstructing cardiac $\mathrm{CT}$ images. It is expected to be used in various ways in human arrhythmia research in the future.

\section{Potential clinical usefulness of cardiac CT-based WT map} The AMBER-WT map has the potential for various clinically: a calculation of ventricular mass, surgical planning for myomectomy for obstructive cardiomyopathy, targeting of papillary muscle VT, and 3D distance measurement between epicardial mapping site and closest endocardial points at the LV summit. The development of various ablation technologies, including irrigated-tip catheters, has made effective ablation lesion formation possible. However, it is practically impossible to create transmural ablation lesions on thick ventricles during VT ablation procedures [31]. Epicardial mapping and ablation techniques have been developed and used for this reason [32], but these techniques involve risks of damaging coronary arteries or phrenic nerves, pericarditis, and bleeding. Ablation energy delivery is also less efficient in epicardial ablation than endocardial ablation [33]. Estimation of the LVWT of epicardial target sites using a 3D thickness map aids in decision-making regarding epicardial ablation or endocardial ablation.

WT maps are expected to be more useful during AF catheter ablation procedures. Recent new technologies, such as high power short duration ablation, contact force monitoring, ablation index, lesion index, and one-shot balloon PVI (cryoballoon and LASER balloon), have been applied in AF catheter ablation. Although these updated technologies may overcome operator-dependent variation, patient factors including local atrial WT are not considered during the procedure. Transmural ablation lesion formation plays a significant role in autonomic modulation as well as long-lasting permanent CPVI [34, 35]. In contrast, excessively high dose energy delivery carries a potential risk of collateral damage or PV stenosis because the atrial wall is thin. Therefore, energy dosing or titration according to LAWT may be used for more effective and safer AF ablation. In this study, we demonstrated that real-time monitoring with a LAWT map and RF energy titration are possible during CPVI procedures. And clinical RCT for RF energy dosing based on LAWT map is expected to begin soon (UTMOST trial, clinical trials.gov NCT 03912324).

\section{Study limitations}

This study had several limitations. During CT segmentation, WT measurements may be inaccurate in areas where cardiac structures are folded or contact extracardiac tissue with similar or overlapping HU. Because the heart is a dynamic structure, local WT can change drastically with systolic and diastolic phases. Although WT was measured after ECG gating, there is a limitation in standardizing WT in arrhythmia states. Hemodynamic status or cardiac chamber pressure may affect WT, but the timing of CT imaging differs from that of echocardiography. The accuracy of WT measurements may deteriorate around regions where WT changes abruptly or around a prominently protruding structure.

\section{Conclusion}

We developed and verified an AMBER 3D-cardiac thickness map measured by cardiac CT images for LAWT and LVWT, and tested its feasibility for RF energy titration during clinical catheter ablation.

\section{Abbreviations \\ WT: Wall thickness; CT: Computed tomogram; LA: Left atrial; LV: Left ven- tricular; HU: Hounsfield units; AF: Atrial fibrillation; RF: Radiofrequency; CAD: Computer-aided design; CPVI: Circumferential pulmonary vein isolation.}

\section{Acknowledgements}

We would like to thank Mr. John Martin for his linguistic assistance and Mr. Jung Kee Lee and Ms. Hye-Rin Ahn for their technical assistance.

\section{Authors' contributions}

O-SK, H-NP, and JL wrote the manuscript. O-SK and H-NP designed experiments and software architecture. O-SK and JL implemented the AMBER software. O-SK, JL, and SL performed experiments. O-SK, JL, SL, and H-JH conducted validation experiments. JL and J-WP contributed to the statistical analysis. S-HY contributed to classifying computed tomogram data. IH contributed to the generation of a voltage map. HTY, T-HK, J-SU, BJ, M-HL, and H-NP contributed to the collection of clinical information and computed tomogram data. All authors read and approved the final manuscript.

\section{Funding \\ This work was supported by grants KHRS 2018-1 from the Korean Heart Rhythm Society.}

\section{Availability of data and materials \\ Available on request.}

\section{Ethics approval and consent to participate}

The study protocol adhered to the Declaration of Helsinki and was approved by the Institutional Review Board of the Yonsei University Health System. All patients provided written informed consent (ClinicalTrials.gov; NCT 02176616).

\section{Consent for publication}

All authors agree with publication of the manuscript.

\section{Competing interests}

The authors declare that they have no competing interest.

Received: 26 May 2020 Accepted: 24 July 2020

Published online: 14 August 2020 


\section{References}

1. Song J-S, Kim J, Lim B, Lee Y-S, Hwang M, Joung B, et al. Pro-arrhythmogenic effects of heterogeneous tissue curvature-a suggestion for role of left atrial appendage in atrial fibrillation. Circ J. 2019;83(1):32-40.

2. Karim R, Blake L-E, Inoue J, Tao Q, Jia S, Housden RJ, et al. Algorithms for left atrial wall segmentation and thickness - evaluation on an opensource CT and MRI image database. Med Image Anal. 2018:50:36-53.

3. Valencia Pérez TA, Hernández López JM, Moreno-Barbosa E, de Celis Alonso B, Palomino Merino MR, Castaño Meneses VM. Efficient CT image reconstruction in a GPU parallel environment. Tomography. 2020;6(1):44-53.

4. Bishop M, Rajani R, Plank G, Gaddum N, Carr-White G, Wright M, et al. Three-dimensional atrial wall thickness maps to inform catheter ablation procedures for atrial fibrillation. Europace. 2016;18(3):376-83.

5. Liao P-S, Chen T-S, Chung P-C. A fast algorithm for multilevel thresholding. J Inf Sci Eng. 2001;17(5):713-27.

6. Beinart ROY, Abbara S, Blum A, Ferencik M, Heist K, Ruskin J, et al. Left atrial wall thickness variability measured by CT scans in patients undergoing pulmonary vein isolation. J Cardiovasc Electrophysiol. 2011;22(11):1232-6.

7. Dewland TA, Wintermark MAX, Vaysman A, Smith LM, Tong E, Vitting-

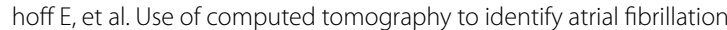
associated differences in left atrial wall thickness and density. Pacing Clin Electrophysiol. 2013;36(1):55-62.

8. Hall B, Jeevanantham V, Simon R, Filippone J, Vorobiof G, Daubert J. Variation in left atrial transmural wall thickness at sites commonly targeted for ablation of atrial fibrillation. J Interv Cardiac Electrophysiol. 2006;17(2):127-32.

9. Hayashi H, Hayashi M, Miyauchi Y, Takahashi K, Uetake S, Tsuboi I, et al. Left atrial wall thickness and outcomes of catheter ablation for atrial fibrillation in patients with hypertrophic cardiomyopathy. J Interv Cardiac Electrophysiol. 2014;40(2):153-60.

10. Hoffmeister PS, Chaudhry GM, Mendel J, Almasry I, Tahir S, Marchese T, et al. Evaluation of left atrial and posterior mediastinal anatomy by multidetector helical computed tomography imaging: relevance to ablation. J Interv Cardiac Electrophysiol. 2007;18(3):217-23.

11. Imada M, Funabashi N, Asano M, Uehara M, Ueda M, Komuro I. Anatomical remodeling of left atria in subjects with chronic and paroxysmal atrial fibrillation evaluated by multislice computed tomography. Int J Cardiol. 2007:119(3):384-8.

12. Inoue J, Skanes AC, Gula LJ, Drangova M. Effect of left atrial wall thickness on radiofrequency ablation success. J Cardiovasc Electrophysiol. 2016;27(11):1298-303.

13. Nakamura K, Funabashi N, Uehara M, Ueda M, Murayama T, Takaoka H, et al. Left atrial wall thickness in paroxysmal atrial fibrillation by multisliceCT is initial marker of structural remodeling and predictor of transition from paroxysmal to chronic form. Int J Cardiol. 2011;148(2):139-47.

14. Pan N-H, Tsao H-M, Chang N-C, Chen Y-J, Chen S-A. Aging dilates atrium and pulmonary veins: implications for the genesis of atrial fibrillation. Chest. 2008;133(1):190-6.

15. Platonov PG, Ivanov V, Ho SY, Mitrofanova L. Left atrial posterior wall thickness in patients with and without atrial fibrillation: data from 298 consecutive autopsies. J Cardiovasc Electrophysiol. 2008:19(7):689-92.

16. Takahashi K, Okumura Y, Watanabe I, Nagashima K, Sonoda K, Sasaki N, et al. Relation between left atrial wall thickness in patients with atrial fibrillation and intracardiac electrogram characteristics and ATP-provoked dormant pulmonary vein conduction. J Cardiovasc Electrophysiol. 2015;26(6):597-605.

17. Yen Ho S, Sanchez-Quintana D, Cabrera JA, Anderson RH. Anatomy of the left atrium. J Cardiovasc Electrophysiol. 1999;10(11):1525-33.

18. Lim B, Hwang M, Song J-S, Ryu A-J, Joung B, Shim EB, et al. Effectiveness of atrial fibrillation rotor ablation is dependent on conduction velocity: an in silico 3-dimensional modeling study. PLOS ONE. 2017:12(12):e0190398-e.
19. Lim B, Kim J, Hwang M, Song J-S, Lee JK, Yu H-T, et al. In situ procedure for high-efficiency computational modeling of atrial fibrillation reflecting personal anatomy, fiber orientation, fibrosis, and electrophysiology. Sci Rep. 2020;10(1):2417

20. Kinno M, Nagpal P, Horgan S, Waller AH. Comparison of echocardiography, cardiac magnetic resonance, and computed tomographic imaging for the evaluation of left ventricular myocardial function: part 1 (global assessment). Curr Cardiol Rep. 2017;19(1):9.

21. Mitchell C, Rahko PS, Blauwet LA, Canaday B, Finstuen JA, Foster MC, et al. Guidelines for performing a comprehensive transthoracic echocardiographic examination in adults: recommendations from the american society of echocardiography. J Am Soc Echocardiogr. 2019;32(1):1-64.

22. Borlich M, Iden L, Kuhnhardt K, Paetsch I, Hindricks G, Sommer P. 3D Mapping for PVI-geometry, image integration and incorporation of contact force into work flow. J Atr Fibrillation. 2018;10(6):1795.

23. Opthof T, Janse MJ, Meijborg VMF, Cinca J, Rosen MR, Coronel R. Dispersion in ventricular repolarization in the human, canine and porcine heart. Prog Biophys Mol Biol. 2016;120(1):222-35

24. Swanson D, Panescu D. Intra-operative RF ablation in the atria. Conf Proc IEEE Eng Med Biol Soc. 2004:2004:5430-2.

25. Dunn FG, Pringle SD. Sudden cardiac death, ventricular arrhythmias and hypertensive left ventricular hypertrophy. J Hypertens. 1993;11(10):1003-10

26. Rodríguez-Sinovas A, Cinca J, Tapias A, Armadans L, Tresànchez M, SolerSoler J. Lack of evidence of M-cells in porcine left ventricular myocardium. Cardiovasc Res. 1997;33(2):307-13.

27. Hwang C, Chen P-S. Ligament of Marshall: why it is important for atrial fibrillation ablation. Heart Rhythm. 2009;6(12):S35-40.

28. de Groot Natasja MS, Houben Richard PM, Smeets Joep L, Boersma E, Schotten U, Schalij Martin J, et al. Electropathological substrate of longstanding persistent atrial fibrillation in patients with structural heart disease. Circulation. 2010;122(17):1674-82.

29. Hansen BJ, Zhao J, Csepe TA, Moore BT, Li N, Jayne LA, et al. Atrial fibrillation driven by micro-anatomic intramural re-entry revealed by simultaneous sub-epicardial and sub-endocardial optical mapping in explanted human hearts. Eur Heart J. 2015:36(35):2390-401.

30. Parameswaran R, Teuwen CP, Watts T, Nalliah CJ, Royse A, Goldblatt J, et al. Functional atrial endocardial-epicardial dissociation in patients with structural heart disease undergoing cardiac surgery. JACC Clin Electrophysiol. 2020;6(1):34.

31. Wilber DJ. Ablation of the epicardial substrate in ventricular tachycardia associated with structural heart disease. J Am Coll Cardiol. 2014;63(14):1427

32. Sosa E, Scanavacca M, D'Avila A, Pilleggi E. A new technique to perform epicardial mapping in the electrophysiology laboratory. J Cardiovasc Electrophysiol. 1996;7(6):531-6.

33. Sacher F, Wright M, Derval N, Denis A, Ramoul K, Roten L, et al. Endocardial versus epicardial ventricular radiofrequency ablation. Circ Arrhythm Electrophysiol. 2013:6(1):144-50.

34. Jin MN, Lim B, Yu Hee T, Kim TH, Uhm JS, Joung B, et al. Long-term outcome of additional superior vena cava to septal linear ablation in catheter ablation of atrial fibrillation. J Am Heart Assoc. 2019;8(22):e013985.

35. Kang K-W, Kim TH, Park J, Uhm JS, Joung B, Hwang C, et al. Long-term changes in heart rate variability after radiofrequency catheter ablation for atrial fibrillation: 1-year follow-up study with irrigation tip catheter. J Cardiovasc Electrophysiol. 2014;25(7):693-700.

\section{Publisher's Note}

Springer Nature remains neutral with regard to jurisdictional claims in published maps and institutional affiliations. 